REVISTA DE GEOCIÊNCIAS DO NORDESTE

Northeast Geosciences Journal

v. $6, n^{\circ} 2(2020)$

https://doi.org/10.21680/2447-3359.2020v6n2ID20843

ISSN: $2447-3359$

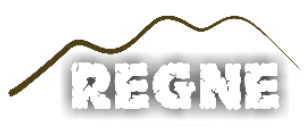

\title{
O AVANÇO DO USO DE FERRAMENTAS GEOESPACIAIS NA GESTÃO DE ÁREAS ÚMIDAS CONTINENTAIS
}

\section{Isadora Taborda Silva ${ }^{1}$; Dhonatan Diego Pessi ${ }^{2}$; Eliane Guaraldo ${ }^{3}$; Antônio Conceição Paranhos Filho $^{4}$}

${ }^{1}$ Mestre em Recursos Naturais, Programa de Pós-Graduação em Recursos Naturais, Universidade Federal de Mato Grosso do Sul (UFMS), Campo Grande/MS, Brasil.

ORCID: https://orcid.org/0000-0001-9053-4329

Email: isah.taborda@gmail.com

${ }^{2}$ Doutorando em Tecnologias Ambientais, Programa de PósGraduação em Tecnolofias Ambientais, Universidade Federal de Mato Grosso do Sul (UFMS), Campo Grande/MS, Brasil.

ORCID: https://orcid.org/0000-0003-0781-785X

Email: dhonatan.pessi@gmail.com

${ }^{3}$ Pós-Doutora em Gestão Urbana, Departamento de Estruturas e Construção Civil, Universidade Federal de Mato Grosso do Sul (UFMS), Campo Grande/MS, Brasil.

ORCID: https://orcid.org/0000-0003-2526-1293

Email: eliane.guaraldo@ufms.br

${ }^{4}$ Doutor em Geologia Ambiental, Faculdade de Engenharias, Arquitetura e Urbanismo e Geografia, Universidade Federal de Mato Grosso do Sul (UFMS), Campo Grande/MS, Brasil.

ORCID: $\underline{\text { https://orcid.org/0000-0002-9838-5337 }}$

Email: toniparanhos@gmail.com

\section{Resumo}

As áreas úmidas continentais são ecossistemas fundamentais para a fauna, flora e o bem-estar da humanidade. A compreensão deste sistema é substancial para a manutenção de seu equilíbrio e reduzir possíveis impactos antrópicos. Essa pesquisa objetiva levantar o progresso das publicações científicas sobre áreas úmidas continentais que utilizem geotecnologias para análise do uso e cobertura do solo. Os principais artigos se referiam a duas vertentes: uma sobre a criação, atualização e aperfeiçoamento de técnicas, métodos e bancos de dados, e a outra no tocante ao monitoramento e avaliação de possíveis impactos. Foram localizadas 2.157 publicações desde a primeira publicação em 1977 até a última no fim de 2019. O conjunto apresentou em sua maioria resultados no formato artigo, com um total de $71,74 \%$ (1.548) das publicações, seguido de $22,97 \%$ (495) de anais de evento, $1,86 \%$ (40) de capítulos de livros, 1,72\% (37) de artigos de revisão, e por fim, $1,72 \%$ (37) de outros tipos de documentos. As pesquisas acerca do uso e cobertura do solo em áreas úmidas com geotecnologias têm mantido um crescimento anual quase constante com uma média de citação considerável e crescimento exponencial que indica uma intensificação na discussão sobre o tema.

Palavras-chave: Sensoriamento remoto; Áreas alagadas; Softwares de geoprocessamento.

\section{THE ADVANCE OF THE USE OF GEOSPATIAL TOOLS IN THE MANAGEMENT OF CONTINENTAL WETLANDS}

\begin{abstract}
Continental wetlands are fundamental ecosystems for fauna, flora and the well-being of humanity. The understanding of this system is substantial for maintaining its balance and reducing possible anthropic impacts. This research aims to survey the progress of scientific publications on continental wetlands that use geotechnologies to analyze land use and cover. The main articles referred to two aspects: one about the creation, updating and improvement of techniques, methods and databases, and the other regarding the monitoring and evaluation of possible impacts. 2157 publications were found from the first publication in 1977 to the last one at the end of 2019. The set presented mostly results in article format, with a total of $71.74 \%$ (1548) of the publications, followed by $22.97 \%$ (495) of event annals, $1.86 \%$ (40) of book chapters, $1.72 \%$ (37) of review articles, and finally, $1.72 \%$ (37) of other types of documents. Research on land use and land cover in humid areas with geotechnologies has maintained an almost constant annual growth with a considerable citation average and exponential growth that indicates an intensification in the discussion on the topic.
\end{abstract}

Keywords: Remote sensing; flooded áreas; geoprocessing software.

\section{EL AVANCE DEL USO DE HERRAMIENTAS GEOESPACIALES EM EL MANEJO DE HUMEDALES CONTINENTALES}

\section{Resumen}

Los humedales continentales son ecosistemas fundamentales para la fauna, la flora y el bienestar de la humanidad. La comprensión de este sistema es sustancial para mantener su equilibrio y reducir 
los posibles impactos antrópicos. Esta investigación tiene como objetivo estudiar el progreso de las publicaciones científicas sobre humedales continentales que utilizan geotecnologías para analizar el uso y la cobertura de la tierra. Los artículos principales se referían a dos aspectos: uno sobre la creación, actualización y mejora de técnicas, métodos y bases de datos, y otro sobre el monitoreo y la evaluación de posibles impactos. Se encontraron 2157 publicaciones desde la primera publicación en 1977 hasta la última a fines de 2019. El conjunto presentó principalmente resultados en formato de artículo, con un total de $71.74 \%$ (1548) de las publicaciones, seguido por $22.97 \%$ ( 495 ) de anales de eventos, $1.86 \%$ (40) de capítulos de libros, $1.72 \%$ (37) de artículos de revisión, y finalmente, $1.72 \%$ (37) de otros tipos de documentos. La investigación sobre el uso de la tierra y la cobertura de la tierra en áreas húmedas con geotecnologías ha mantenido un crecimiento anual casi constante con un promedio de citas considerable y un crecimiento exponencial que indica una intensificación en la discusión sobre el tema.

Palabras-clave: Teledetección; Áreas inundadas; Software de geoprocesamiento.

\section{INTRODUÇÃO}

As áreas úmidas são zonas onde a água é o principal fator de controle ambiental da fauna e flora associadas. Apesar de sua importância, estima-se que mais de $70 \%$ das áreas úmidas globais já tenham sido destruídas ou foram comprometidas desde 1900 (GARDNER et al., 2015). Logo, estudos nestas áreas são de grande importância para protegê-las e reduzir possíveis alterações. Porém são áreas de difícil monitoramento por métodos convencionais devido à sua relativa inacessibilidade e dinâmica sazonal (HEWES, 1951; LEE; LUNETTA, 1996; MITCH; GOSSELINK, 2007). Estas dificuldades fazem do sensoriamento remoto uma forma proficiente de análise (FRANÇA; SANO, 2011; GUO et al., 2017). Sua utilização para estudos ambientais é consagrada na literatura, possibilitando análises e elaborações de mapeamentos de vegetação e de impactos ambientais, dentre tantos outros.

Sendo assim, muitos trabalhos são produzidos com uso de ferramentas de geoprocessamento, do qual destaca-se o sensoriamento remoto, muitas vezes aliada a visitas de campo com o intuito de avaliar e monitorá-las. No entanto, poucos resumiram o progresso das pesquisas na área. Para preencher esta lacuna, o presente artigo objetiva quantificar as publicações acadêmicas sobre áreas úmidas continentais que utilizam sensoriamento remoto focando em analisar o uso e cobertura do solo. Além disso, é investigada a utilização ou não de softwares livres nas pesquisas.

Neste sentido, a análise bibliométrica é um método eficaz para analisar publicações acadêmicas utilizando técnicas estatísticas e as correlacionando com determinados assuntos. Permite mensurar a performance acadêmica em determinada área da ciência trazendo luz a sua estrutura e padrão de desenvolvimento (ZHANG et al., 2015) e tem sido amplamente aceita por oferecer perspectivas inovadoras na avaliação de tendências em pesquisas através de análise de conteúdo (MAO et al., 2015).

Portanto, o objetivo dessa pesquisa foi quantificar as publicações acadêmicas sobre áreas úmidas continentais que analisem o uso e cobertura do solo com base em ferramentas de sensoriamento remoto para obter uma base do progresso das pesquisas na área.

\section{METODOLOGIA}

A bibliometria e os indicadores bibliométricos, no seu conjunto, são utilizados para avaliar a produtividade dos autores, realizar estudos de citações e avaliar a qualidade dos periódicos científicos (LOPES et al., 2012).

$\mathrm{Na}$ análise bibliométrica os principais indicadores relacionados são as citações de cada publicação, o fator de impacto (FI) dos periódicos e o índice $\mathrm{H}$ ( $h$-index) do pesquisador (WOOD JÚNIOR; DE MEDEIROS COSTA, 2015). Os três foram utilizados nesse estudo para verificar o impacto da produção científica, juntamente com os dados de ano de publicação, país de origem e palavras-chaves dos artigos.

Para representar o universo de estudo foram definidas algumas palavras-chaves apresentadas na Tabela 1. Por fim, para formação da cadeia de caracteres (string) considerou-se os radicais das palavras e suas possíveis variações na literatura. Devido à grande abrangência de estudos em inglês, os termos foram utilizados em língua inglesa.

Tabela 1 - Definição dos termos em inglês a serem utilizados para formação da string, advindos das palavras-chaves em português, e seus respectivos sinônimos encontrados na literatura. Fonte: os autores (2020).

\begin{tabular}{cc|}
\hline TERMOS & SINÔNIMOS EM INGLES \\
\hline Áreas Úmidas Continentais & Wetland \\
\hline Geotecnologias e & Remote Sensing \\
Sensoriamento Remoto & Geotecnolog* \\
& Geotechnolog* \\
& GIS \\
& Geoprocessing* \\
\hline Uso e Cobertura do Solo & Satellite Image* \\
& Land Use \\
& Land Cover \\
& LULC \\
& Soil Cover \\
\hline
\end{tabular}


Por fim, a string principal de busca ficou definida como: Wetland* AND ("Remote sensing" OR Geotecnolog* OR Geotechnolog* OR GIS OR "Satellite Image") AND ("land use" OR "land cover" OR "Soil cover").

As pesquisas foram realizadas em bases indexadas no Portal de Periódicos da Coordenação de Aperfeiçoamento de Pessoal de Nível Superior - CAPES/MEC, cujo acesso é disponibilizado aos acadêmicos através da parceria entre a CAPES e a Universidade
Federal de Mato Grosso do Sul - UFMS. Três grandes Bases de Dados com uso popular no cenário acadêmico foram selecionadas inicialmente: Scopus (ELSEVIER, 2020a), Science Direct (ELSEVIER, 2020b), e Web of Science - Coleção Principal (CLARIVATE ANALYTICS, 2020a). As buscas se limitaram a títulos, resumos e palavras-chave e em documentos publicados até 2019 (Tabela 2).

Tabela 2 - Retorno da string Wetland* AND ("Remote sensing" OR Geotecnolog* OR Geotechnolog* OR GIS OR "Satellite Image") AND ("land use" OR "land cover" OR "Soil cover") nas bases de dados. Fonte: Compilado de Scopus (ELSEVIER,2020a), Science Direct (ELSEVIER,2020b) e Web of Science (CLARIVATE ANALYTICS, 2020a).

BASE DE DADOS

\begin{tabular}{cc}
\hline Science Direct & 373 publicações \\
\hline Web of Science & 1424 publicações \\
Scopus & 2157 publicações \\
\hline
\end{tabular}

Após download dos registros, gravando o conteúdo 'Autor, Título, Fonte e Resumo', estes foram organizados no software livre Mendeley (ELSEVIER, 2020c) que gerencia referências permitindo uma abordagem bibliométrica para avaliar o resultado das pesquisas e remover eventuais duplicidades. Posterior a préseleção, verificou-se que as bases de dados Web of Science e Science Direct continham, em sua maioria, publicações já existentes na base Scopus. Sendo assim, definiu-se utilizar apenas esta última como banco de dados chave da pesquisa, usando dados referentes à tipos de documentos, idiomas, áreas do conhecimento abrangidas, palavras-chaves, países dos autores, tipo de acesso e softwares utilizados.

Em sequência, foi utilizado como ferramenta bibliométrica o software livre VOSviewer (VAN ECK; WALTMAN, 2019) que cria redes de co-citações a partir de arquivos de algumas bases de dados com informações sobre bibliografia, citações, resumo e palavras-chaves. $\mathrm{O}$ emprego do programa auxiliou na construção das redes de relações de coautoria entre diferentes países e de reincidências de palavras-chaves.

Após analisados os dados gerais, foram especificados os artigos mais citados para traçar um panorama sobre as principais discussões acerca do tema. Com os 10 artigos mais citados dentro das publicações retornadas, levantou-se para cada um destes o fator de impacto do periódico $(J C R)$ e o $H$-index do autor, pois considerou-se que a influência do autor também poderia induzir na disseminação do artigo. Como havia poucas informações do $H$ index dos autores na base da Scopus, foram considerados os dados encontrados na base Google Acadêmico, fonte de busca construída pelos próprios pesquisadores considerada então confiável e válida para divulgar pesquisas e consultar dados. Para cada artigo selecionou-se o maior $H$-index entre os autores, bem como as citações que constassem na base Scopus (ELSEVIER, 2020a) até as buscas em janeiro de 2020.

Discutido os principais artigos no tema ao longo dos anos, buscou-se uma linha de tendências das publicações nos últimos quatro anos (2016 a 2019). Novamente, foram levantados os artigos mais citados nesses anos, bem como o JCR e o maior $H$ index dentre os encontrados na base do Google Acadêmico (GOOGLE, 2020).

Posteriormente, buscou-se saber quais destes faziam menção ao programa empregado na pesquisa e quantos eram livres ou código aberto. Segundo a Free Software Foundation (2019), as vantagens são inúmeras de utilizá-los ao invés dos softwares proprietários (necessitam de compra para sua utilização). Além de não acarretarem custos aos pesquisadores, os que possuem também livre acesso ao código fonte permitem mudanças para se adequarem ao uso. As correções do programa são mais rápidas e com aprimoramentos mais eficientes graças a cultura de divulgação e compartilhamento entre os usuários e os desenvolvedores.

De início refinou-se a string principal com o termo software, pois notou-se que poucos dos artigos citavam quais teriam utilizado. Com os 250 documentos resultantes, foram separados quais utilizavam programas livre ou de código livre aplicando outro refinamento. Os termos utilizados neste segundo refinamento foram: ("Free software" OR "Open source" OR "Open software" OR "Freeware"). Analisou-se então os 18 documentos resultantes deste último afunilamento.

\section{RESULTADOS E DISCUSSÃO}

\subsection{Análises quantitativas}

Ao todo, foram localizadas 2.157 publicações, desde a primeira publicação em 1977 até a última no fim de 2019. Como mostra a Figura 1 o conjunto apresentou em sua maioria resultados no formato artigo, com um total de $71,74 \%$ (1.548) das publicações, seguido de 22,97\% (495) de anais de evento, 1,86\% (40) de capítulos de livros, 1,72\% (37) de artigos de revisão, e por fim, $1,72 \%$ (37) de outros tipos de documentos. Para obter um resultado apurado, foram considerados para a análise apenas artigos científicos e artigos de revisão (1.585 artigos). 


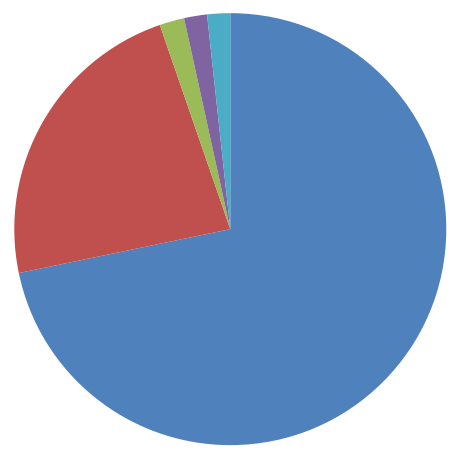

- Artigos $(71,7 \%)$

- Anais de evento (23\%)

- Capítulo de livro $(1,8 \%)$

- Artigo de revisão $(1,7 \%)$

- Outros $(1,7 \%)$

Figura 1 - Gráfico com os formatos dos documentos encontrados na base Scopus, onde a maioria das publicações são no formato Artigo. Fonte: Compilado de Scopus (ELSEVIER,2020a).

$\mathrm{O}$ número de publicações por ano (PA) manteve um crescimento anual quase constante (Figura 2). De 1977 até 1995, manteve-se uma média de 2,74 PA. Após esse período, esta média eleva-se para 15,62 PA até o ano 2003 e depois quadruplica para 65,45 PA de 2004 a 2014. Nos últimos 5 anos, a média cresce novamente, dobra e atinge seu ápice com 137,2 PA. Os três anos mais produtivos estão nesse último período, sendo eles o ano 2019 com 169 PA, seguido dos anos 2018 com 135 PA e 2015 com 132 PA.

Aliado ao crescimento constante de publicações, está o de citações. Ao todo, as 1.585 publicações somam 34.990 citações, com uma média de 22,10 citações por item. Ao comparar o total de citações por ano (CA) com o de PA na Figura 3, verifica-se que as primeiras citações ocorreram logo após o início das publicações, porém começaram a ser expressiva nos anos 2000. Nos últimos 7 anos a quantidade de citações dobraram indo de 2.130 citações/ano em 2013 a 5.494 em 2019, indicando uma intensificação na discussão sobre o tema.

As publicações abrangem 23 áreas da ciência, sendo que um documento pode envolver múltiplas áreas (Figura 4). Dentre elas, Ciências Ambientais destaca-se com quase um terço das publicações e despontando 936 vezes $(34,04 \%)$, seguido das áreas Geociências com 570 publicações (20,73\%) e Ciências Agrárias e Biológicas com 527 vezes $(19,16 \%)$. Juntas, as outras 20 áreas do conhecimento figuram com 717 publicações $(26,07 \%)$.

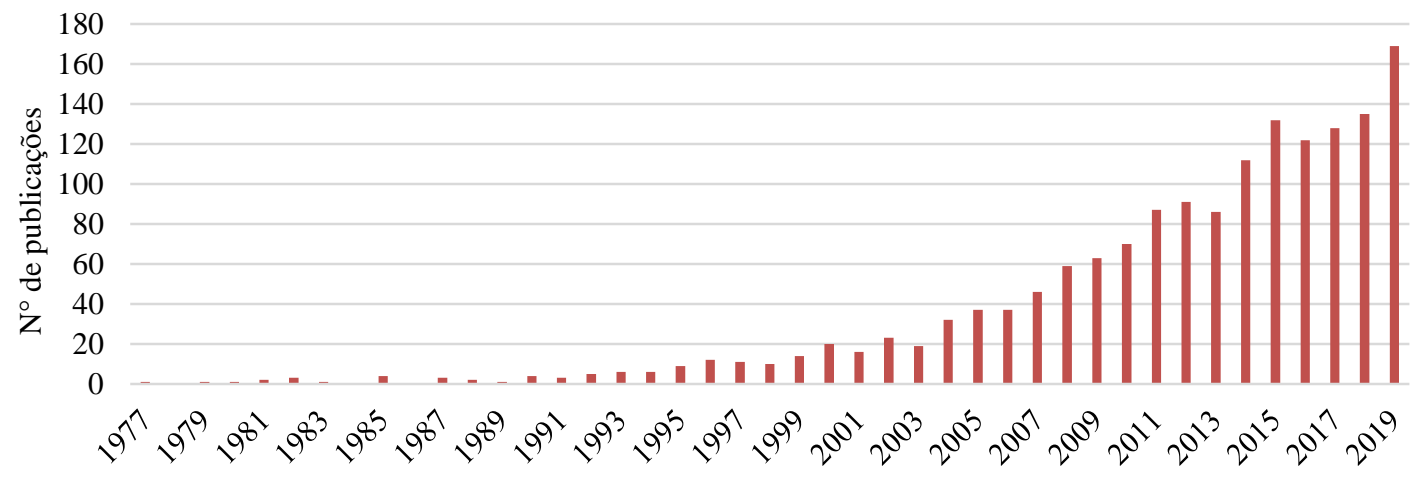

Figura 2 - Gráfico de publicações ao ano demonstrando o crescimento constante nas publicações acerca do tema. As publicações tiveram início em 1977, mas se tornaram constantes na década de 90. Os últimos 5 anos (2015 a 2019) são os mais produtivos e atingiram uma média de 137,2 publicações ao ano. Fonte: Compilado de Scopus (ELSEVIER,202Oa). 


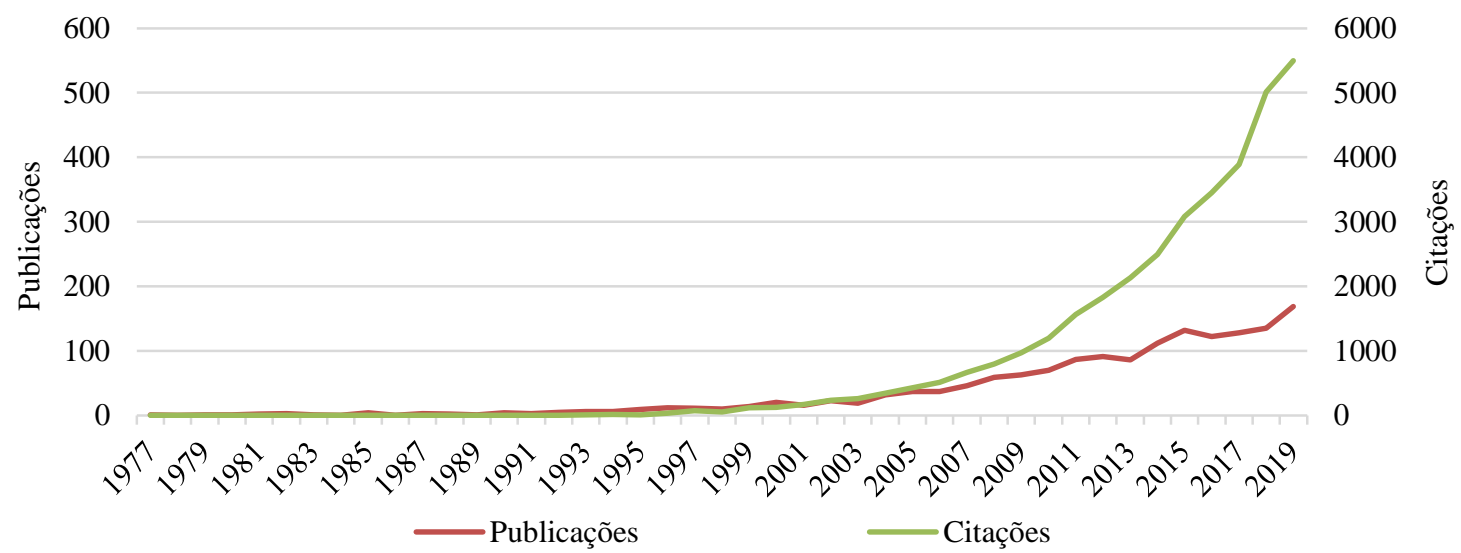

Figura 3 - Gráfico de combinação onde o eixo vertical esquerdo são as quantidades de publicação por ano e o eixo direito de citação por ano. O ápice das citações em 2019 corresponde a um valor em torno de 30 vezes maior que o de publicações no mesmo ano, evidenciando a recorrência do tema. Fonte: Compilado de Scopus (ELSEVIER,2020a).

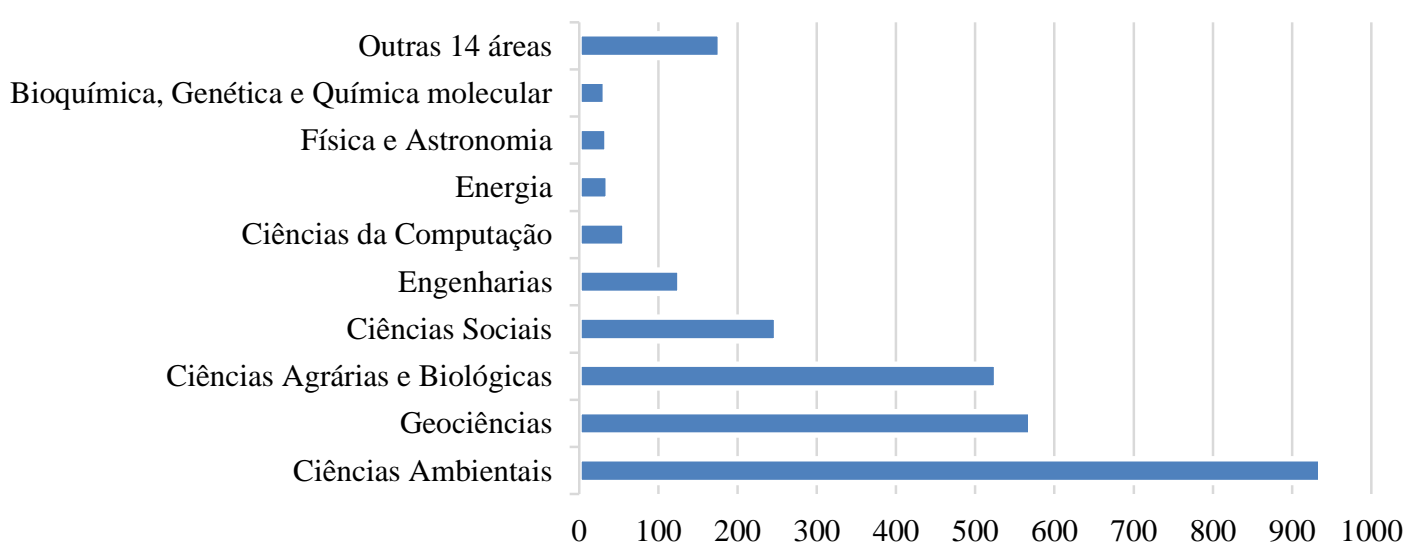

Figura 4 - Gráfico das áreas da ciência que abrangem as publicações. Destaca-se Ciências Ambientais com um terço dos artigos. Fonte: Compilado de Scopus (ELSEVIER,2020a).

Entre os idiomas principais das publicações, conforme o informado pelos autores, o inglês destaca-se com 1.371 publicações (86\%), mesmo essa sendo a língua oficial de apenas 6 dos 15 países com maior quantidade de publicações. O chinês também se sobressai com 185 publicações $(11,6 \%)$ e, em uma quantidade ínfima, constam algumas publicações em outros idiomas como espanhol, francês, alemão, português, arábico, persa, polonês, japonês, russo, eslovaco e húngaro que juntos correspondem a $2,4 \%$ do total (37 artigos).

Somando-se, os 15 países com as maiores quantidades de publicações detêm 75,3\% dos artigos sobre o tema. Os pesquisadores dos Estados Unidos, China e Canadá lideram este ranking quantitativo (Tabela 3 ).

O EUA é o país com maior número de publicações e citações, apresentando o triplo de citações do segundo colocado China, que possui quantidade próxima de publicações. Sendo assim, a média de citações por itens da China configura como a menor entre as quatro primeiras, sendo superior apenas as médias da Índia e do
Irã. Ainda analisando as médias, o Reino Unido $\left(6^{\circ}\right)$, o Canadá $\left(3^{\circ}\right)$ e a Alemanha $\left(4^{\circ}\right)$ são os países que apresentam a maior média de citação por item indicando serem os países com publicações mais influentes. Com 36 documentos publicados, o Brasil encontra-se em $10^{\circ}$ lugar, empatado com os Países Baixos quanto ao número de publicações, mas com a média de citações menor que este.

Utilizando a análise em redes, investigou-se a relação de cooperação internacional dos autores. Para a criação deste estudo gráfico, foram selecionados os 35 países com 10 ou mais publicações. Para favorecer a visualização também ficou estipulado que estes países deveriam, no mínimo, possuir três conexões de coautoria, sendo então excluídos a Turquia (coautoria apenas com os EUA), a Argentina (relação com os EUA e a Espanha), a Grécia (relação com a Suécia) e a Coréia do Sul (sem conexões). Sendo assim, 31 de 109 países (28\%) atenderam os requisitos resultando na Figura 5. 
Tabela 3 - Os quinze países com maior quantidade de publicação. Estados Unidos e China lideram o grupo, possuindo juntos em torno de $50 \%$ do total. Fonte: Compilado de Scopus (ELSEVIER, 2020a).

\begin{tabular}{|ccccc}
\hline PAÍs & $\begin{array}{c}\text { TOTAL DE } \\
\text { PUBLICAÇÕES }\end{array}$ & $\begin{array}{c}\text { TOTAL DE } \\
\text { PUBLICAÇÕES } \\
(\%)\end{array}$ & $\begin{array}{c}\text { TOTAL } \\
\text { CITAÇÕES }\end{array}$ & $\begin{array}{c}\text { MÉDIA DE } \\
\text { CITAÇÃO/ITEM }\end{array}$ \\
\hline Estados Unidos & 507 & $24,3 \%$ & 20012 & 39,47 \\
\hline China & 493 & $23,6 \%$ & 6735 & 13,66 \\
\hline Canadá & 108 & $5,2 \%$ & 4919 & 45,55 \\
\hline Alemanha & 71 & $3,4 \%$ & 3193 & 44,97 \\
\hline Índia & 58 & $2,8 \%$ & 613 & 10,57 \\
\hline Reino Unido & 49 & $2,3 \%$ & 2713 & 55,37 \\
\hline Austrália & 41 & $2,0 \%$ & 863 & 21,05 \\
\hline França & 38 & $1,8 \%$ & 689 & 18,13 \\
\hline Países Baixos & 36 & $1,7 \%$ & 1180 & 32,78 \\
\hline Brasil & 36 & $1,7 \%$ & 677 & 18,81 \\
Turquia & 33 & $1,6 \%$ & 1139 & 34,52 \\
\hline Japão & 29 & $1,4 \%$ & 1004 & 34,62 \\
\hline Espanha & 27 & $1,3 \%$ & 414 & 15,33 \\
Irã & 25 & $1,2 \%$ & 81 & 3,24 \\
\hline México & 23 & $1,1 \%$ & 601 & 26,13 \\
\hline Demais 84 países & 516 & $24,7 \%$ & - & - \\
\hline
\end{tabular}

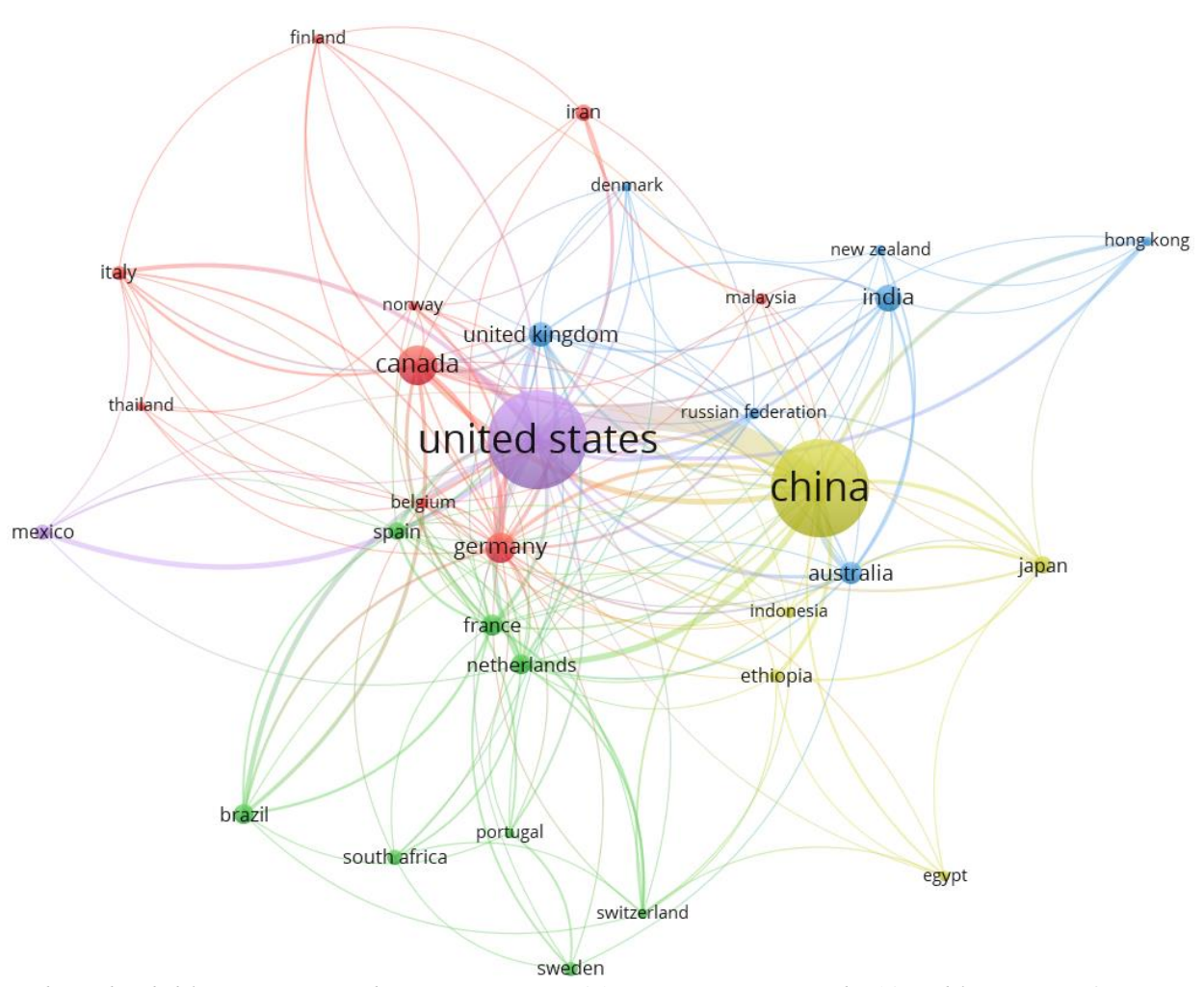

Figura 5 - Análise de redes bibliométricas onde os nós são os 31 países com mais de 10 publicações e 2 coautorias e as ligações representam a cooperação entre eles. A Alemanha se destaca com cooperação com 27 países, sendo eles os países com mais coautoria internacional. Elaborado no VOSviewer (VAN ECK e WALTMAN, 2020) com dados compilados de Scopus (ELSEVIER,2020a). 
Os nós correspondem aos países, sendo a dimensão definida pela sua quantidade total de publicações. As arestas de ligação entre eles se referem às relações de coautoria, onde quanto mais espesso mais há coautoria entre os países. Os clusters foram definidos pelo programa de acordo com a afinidade de publicação entre os países, separando-os em grupos de 5 cores.

Com maior quantidade de relações de cooperação, a Alemanha destaca-se relacionando com 27 dos 31 países analisados, entre eles estão os EUA, a China e o Canadá, países com maior quantidade de publicações. Também se associa ao Reino Unido, que junto com o Canadá, formam o trio com maior média de citações por item demonstrando cooperação entre os países com as publicações mais influentes.

Os pesquisadores dos EUA coescrevem com autores de 26 países, sendo o maior fluxo de cooperação com a China, indicado pelo link mais espesso. Entre as outras conexões, destaca-se as com Canadá, país que apresenta o segundo maior fluxo cooperativo, seguidos de México, Reino Unido, Alemanha, Países Baixos, Brasil e França.
O Brasil mantém oito conexões de coautoria internacional coescrevendo com autores dos EUA, Canadá, Alemanha, França, Espanha, Suíça, Portugal e China. O maior fluxo é com os EUA, porém a diferença entre os fluxos é irrisória. Dentre os grupos de pesquisas brasileiros que se destacam estão o Instituto de Pesquisas Nacionais Espaciais (INPE) com 10 pesquisas, a Universidade de São Paulo (USP) com 4 e com 2 publicações as Universidades Federais do Rio de Janeiro (UFRJ), de Mato Grosso (UFMT), do Mato Grosso do Sul (UFMS), do Pará (UFPA), do Rio Grande do Norte (UFRN) e as Universidades de Brasília (UNB) e a Universidade Estadual Paulista (UNESP).

Ainda utilizando o sistema de análises de redes, foram elencadas as palavras-chaves que estariam em maior evidência. Foram excluídos deste grupo as palavras utilizadas na string (wetland, remote sensing, land use, GIS, land cover, satellite image) por serem termos da busca e estarem presentes na maioria dos artigos como palavras-chaves. Por fim, selecionou-se para representar a rede as que repetiam no mínimo 80 vezes, retornando 27 palavras das 10.045 restantes (Figura 6).

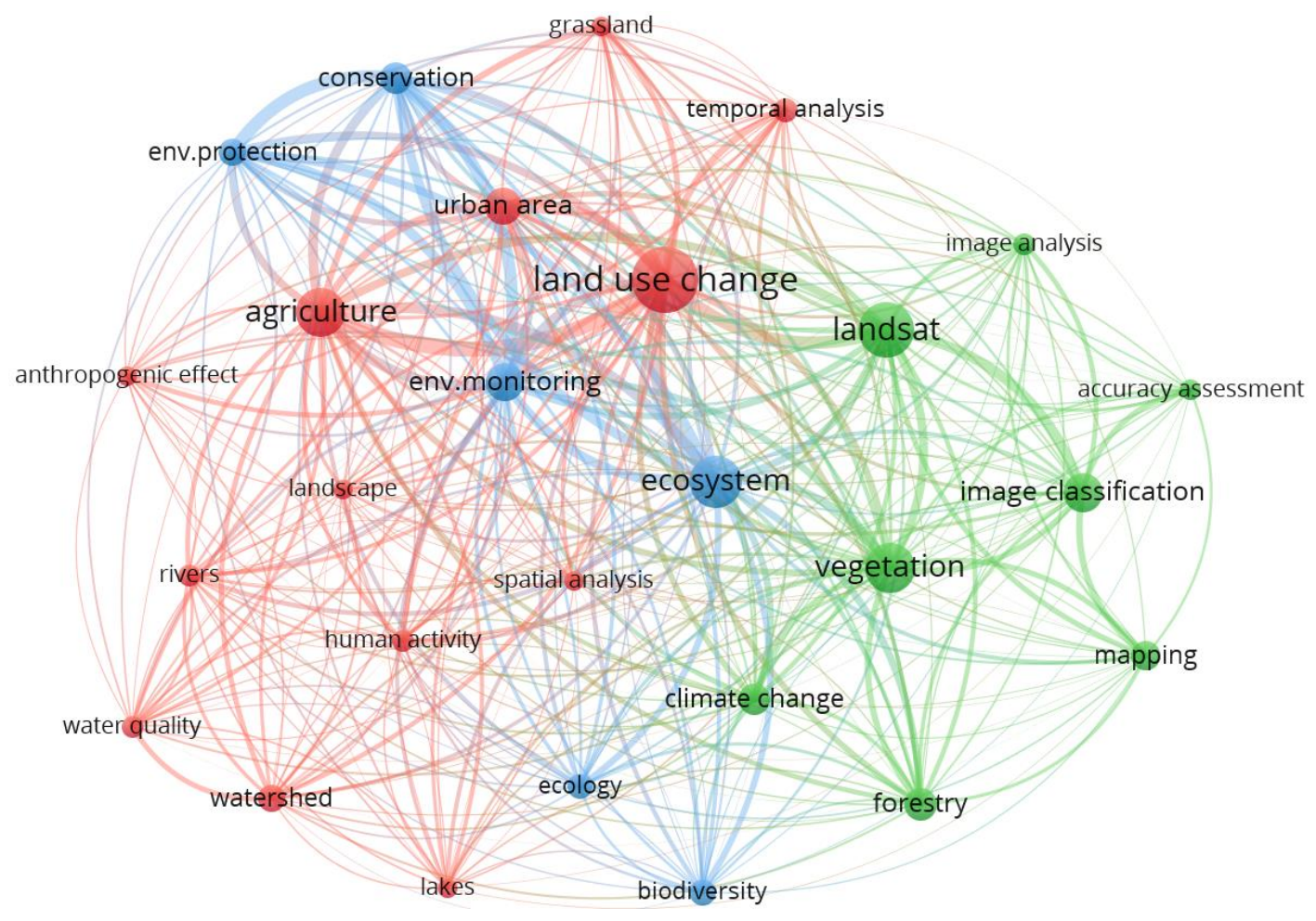

Figura 6 - Análise de redes com as 27 principais palavras-chaves, excetuados os termos da string. Ao centro figuram as mais recorrentes: Land use change, Landsat, ecosystem e agriculture. Fonte: Elaborado no VOSviewer (VAN ECK e WALTMAN, 2019) com dados compilados de Scopus (ELSEVIER,2020a).

Presente em 352 artigos e figurando como elemento central da rede está o termo 'Mudança no Uso do Solo'. Conecta-se principalmente com 'Agricultura', este fazendo ligação com 'Ecossistema' e 'Monitoramento Ambiental', indicando que no tema apresenta-se uma grande parcela de artigos relativos ao avanço da área agrícola sob os recursos nativos e aos monitoramentos de possíveis impactos dessas áreas. A grande reincidência do termo 'Landsat' (programa de imagens de satélites do governo americano) o faz ser, possivelmente, a principal imagem de satélite utilizada, principalmente aliado à 
'Mudança de Uso do Solo', 'Classificação de Imagens' e 'Vegetação'. 'Ecossistema' com 'Monitoramento Ambiental' e 'Mudanças no clima', também configuram uma ligação frequente, assim como 'Proteção Ambiental' com o 'Monitoramento Ambiental', 'Conservação' e 'Agricultura'.

\subsection{Principais publicações do tema}

A quantidade de citações são referências que auxiliam a encontrar trabalhos com maior visibilidade e credibilidade. Para uma análise histórica do tema foram selecionadas as 10 publicações mais citadas (Tabela 4).

Como consta na tabela, entre esses artigos foram analisados também o Fator de Impacto do Periódico (JCR) e o H-index do autor, pois considera-se que estes podem influenciar na disseminação do artigo. Por falta de informação de todos os primeiros autores, foi considerado o maior $H$-index entre os que constasse na plataforma do Google Acadêmico (GOOGLE, 2020).

O periódico "Nature" tem o JCR maior entre as dez e a publicação mais recente dentre as demais (2016). "Remote sensing of environment" possui o segundo JCR mais alto entre os periódicos e figura com quatro publicações na lista. Em média, o $J C R$ das revistas é de 9,296 e o $h$-index dos autores de 68,2. Notase que, de maneira geral, em cada publicação há ao menos um autor com quantidade de publicações e H-index consideravelmente maior em relação aos demais, possivelmente figurando como orientador ou coordenador desses grupos de pesquisas.

Tabela 4 - Os dez artigos mais citados com o número de citações, o maior h-index entre os autores e fator de impacto do periódico. Fonte: Compilado de Scopus (ELSEVIER, 2020a), Google Acadêmico (GOOGLE, 2020) e InCites Journal Citation Reports (CLARIVATE ANALYTICS,2020b).

\section{ARTIGO}

FRIEDL, M. A. et al. MODIS Collection 5 global land cover: Algorithm refinements and characterization of new datasets.

Remote sensing of Environment, v. 114, n. 1, p. 168-182, 2010.

LEHNER, B.; DÖLL, P. Development and validation of a global database of lakes, reservoirs and wetlands. Journal of Hydrology, v. 296, n. 1-4, p. 1-22, 2004.

OZESMI, S. L.; BAUER, M. E. Satellite remote sensing of wetlands. Wetlands ecology and management, v. 10, n. 5, p. 381-402, 2002.

ROTH, N. E.; ALLAN, J. D.; ERICKSON, D. L. Landscape influences on stream biotic integrity assessed at multiple spatial scales. Landscape ecology, v. 11, n. 3, p. 141-156, 1996.

OLOFSSON, P.; FOODY, G.; HEROLD. M.; STEHMAN; S.V.; WOODCOCK, C.E.;

WULDER, M.A. Good practices for estimating area and assessing accuracy of land change.

Remote Sensing of Environment, v. 148, p. 42-57, 2014.

YUAN, F.; SAWAYA, K.E.; LOEFFELHOZ, B.C.; BAUER, M.E.

Land cover classification and change analysis of the Twin Cities Metropolitan Area by multitemporal Landsat remote sensing. Remote sensing of Environment, v. 98, n. 2-3, p. 317-328, 2005.

WALKER, D. A. et al. The circumpolar Arctic vegetation map.

Journal of Vegetation Science, v. 16, n. 3, p. 267-282, 2005.

DEWAN, A. M.; YAMAGUCHI, Y. Land use and land cover change in Greater Dhaka, Bangladesh: Using remote sensing to promote sustainable urbanization. Applied geography, v. 29, p. 390-401, 2009.

PAUSTIAN, K.; LEHMANN, J.; OGLE, S.B; REAY, D.; ROBERTSON, G.P.; SMITH, P. Climate-smart soils (Review).

Nature, v. 532, Issue 7597, p. 49-57, 2016.

KASISCHKE, E. S.; MELACK, J. M.; DOBSON, M.C. The use of imaging radars for ecological applications - a review. Remote sensing of environment, v. 59, n. 2, p. 141-156, 1997.

$\begin{array}{ccc}\begin{array}{c}\text { CIT. } \\ \text { (até Jan.2020) }\end{array} & \text { H-index } & \begin{array}{c}\text { FI } \\ \text { (JCR) }\end{array} \\ 1.494 & 65 & 8.218\end{array}$

616

50

580

64

4.349

564

80

8.218

69

2.944

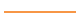


A metade destes artigos possuem apenas autores de um só país (EUA ou Japão). Em quatro deles constam autores de dois países, sendo estes EUA/Canadá, EUA/Alemanha, EUA/Reino Unido e EUA/Turquia. O artigo "The circumpolar Artic vegetation map" é que possui mais rede de colaboração, com 13 autores incluindo autores dos EUA, Alemanha, Islândia, Noruega, Porto Rico e Rússia.

Com mais citações o artigo "MODIS Collection 5 global land cover: Algorithm refinements and characterization of new datasets" foi publicado em 2010, sendo então um dos mais novos da lista. Nele Friedl et al. (2010) descreveram sobre os conjuntos de dados e algoritmos que criaram o MODIS Global Land Cover Type (NASA) e suas alterações até a Coleção 4 e Coleção 5. Concluíram com testes a fim de demonstrar a maior eficácia da última coleção. $\mathrm{O}$ artigo citou as áreas úmidas continentais relacionando-as a casos especiais que são problemáticos e difíceis de mapear, necessitando reduzir o limiar utilizado para classificar os pixels.

Lehner e Döll (2004) apresentaram um novo banco de dados chamado Global Lakes e Wetlands Database (GLDW) focado em três níveis, sendo estes grandes lagos e reservatórios, corpos d'agua menores e áreas úmidas. Para tanto, foi utilizado dados de outros bancos, registros e inventários que possuíam atributos descritivos sobre as áreas, aliado a mapas (analógicos ou digitais) com suas extensões espaciais.

Ozesmi e Bauer (2002) fizeram o primeiro artigo de revisão da lista, comparando métodos de classificações e identificação das melhores técnicas para classificação.

Roth, Allan e Erickson (1996) avaliaram a condição de uma bacia hidrográfica e o impacto ambiental da agricultura na região utilizando os índices IBI (Index of Biotic Integrity) e HI (Habitat Index). Inspecionaram 23 cabeceiras e as classificaram em busca da correlação da integridade dos córregos e qualidade do ambiente conforme a extensão de agricultura, áreas úmidas e florestas.

Olofsson et al. (2014) padronizaram nessa revisão várias recomendações para projetar e implementar métodos de avaliação de precisão e estimativa de área chamadas pelos autores de recomendações de boas práticas.

Yuan et al. (2005) mapearam e analisaram as mudanças de uma área urbana utilizando análise multitemporal de diferentes cidades da área metropolitana de Minnesota de 1986 a 2002 utilizando imagens Landsat. É o primeiro artigo da lista a fazer referência ao software GIS utilizado ERDAS IMAGINE (HEXAGON).

Walker et al. (2005) delinearam as principais unidades de vegetação no Ártico e, utilizando o ArcInfo (ESRI), geraram uma classificação por imagens NOAA VHRR da região.

Com uma série temporal de imagens Landsat, Dewan e Yamaguchi (2009) avaliaram as mudanças de cobertura e uso do solo da expansão urbana de Greater Dhaka/Bangladesh e suas dinâmicas.

Na publicação mais recente, Paustian et al. (2016) tratam de revisão do potencial para mitigar gases de efeito estufa que o solo possui. Destacam pesquisas sobre os gases, resumem práticas e potenciais de mitigação, identificam lacunas nos dados e na compreensão dos mesmo e sugerem novas pesquisas, tecnologias e colaboração.
Por fim, Kasischke et al. (1997) reviram as publicações que utilizaram radar de abertura sintética para a investigação de processos ecossistêmicos e posteriormente as separaram em quatro grandes categorias: classificação e detecção das mudanças na cobertura terrestre; estimativa de biomassas de lenhosas; monitoramento dos momentos de inundação e suas extensões; e monitoramento de outros processos temporalmente dinâmicos.

Sintetizando pode se dizer que as publicações se referem principalmente a duas vertentes: uma sobre a criação, atualização e aperfeiçoamento de técnicas, métodos e bancos de dados, e a outra no tocante ao monitoramento e avaliação de possíveis impactos.

\subsection{Tendências do tema}

Para analisar as tendências do tema, bem como localizar possíveis lacunas, foram analisadas as cinco publicações mais citadas das 554 dos últimos quatro anos da pesquisa (2016 a 2019) (Tabela 5).

A revista "Nature" é o periódico com maior JCR e aqui lidera a lista com o artigo de revisão "Climate-smart soils" de Paustian et al. (2016) que é o $9^{\circ}$ mais citado da análise histórica (Tabela 4). Em sequência, a revista "Proceedings of the National Academy of Sciences of the United States of America" apresenta o segundo maior JCR da lista. No geral, os artigos possuem uma média de 160,6 citações e $J C R$ de 12.341.

A média do $H$-index entre os pesquisadores destaques desses últimos quatro anos é de 53,25 mantendo-se próxima à da análise histórica citada anteriormente de 68,2 . Porém, diferentemente do verificado na análise anterior, notou-se que a maioria dos autores ou não possuíam a informação do $H$-index ou não havia realce significativo entre eles nos grupos de pesquisa. É possível inferir que estes grupos diferem dos anteriores ao apresentar mais pesquisadores influentes do que anteriormente e menos a figura de líder de grupo. Destaca-se que nenhum dos autores do artigo "Assessment of soil erosion change and its relationships with land use/cover change in China from the end of the 1980s to 2010" (WANG et al., 2016) possuíam a informação na plataforma do Google Acadêmico (GOOGLE, 2020). Em relação a nacionalidade e coautoria entre diferentes países, três dos cinco artigos possuem autores de dois países.

Richards e Friess (2016) aplicam combinadamente ferramentas GIS e métodos de sensoriamento remoto para quantificar o desmatamento do mangue no sudoeste asiático dos anos 2000 a 2012. A pesquisa identificou uma perda de $0,18 \%$ ao ano de florestas do mangue. A rápida expansão da agricultura de arroz e as crescentes plantações de óleo de palma são os principais causadores do desmatamento. É o primeiro artigo dentre os citados a tratar especificamente de área úmida.

Em sequência, Ottinger et al. (2016) fazem uma revisão sobre o tema Aquicultura trazendo sua relevância. Abordam os impactos positivos da criação de produtos aquáticos ao evitar perda de áreas úmidas costeira e a poluição de águas e solos. O artigo aponta também para o potencial do sensoriamento remoto para monitorar essas áreas em grande escala.

Tian et al. (2016) apresenta os impactos de longo prazo das áreas costeiras da China através de análise multitemporal de 1985 a 2010. O estudo fornece distribuições espaciais e temporais dessas áreas e analisa fatores a economia costeira, o crescimento 
populacional e a urbanização das regiões que estão diretamente conectadas a elas. O contínuo uso de longa escala trouxe inúmeros efeitos ambientais negativos para a região e indica potencial para futuros desastres relacionados a inundações costeiras.

Por fim, Wang et al. (2016) utiliza uma abordagem empírica para analisar erosão do solo em relação ao uso e mudança do solo na China entre 1980 a 2010. A erosão hídrica é encontrada mais amplamente distribuída no país, seguida da eólica e por congelamento e degelo. A maior parte das alterações causadas pela erosão foram concentradas nas regiões montanhosas do sul da China e nas regiões do norte que possuem ecossistemas mais frágeis, como o Loess Plateau, os desertos de $\mathrm{Mu}$ Us, Hunshandake e Horqin, a área úmida de Xianghai e a bacia do rio Tarim.

Tabela 5 - Os cinco artigos mais citados de 2016 a 2019 com o número de citações, o maior h-index entre os autores e fator de impacto do periódico. Fonte: Compilado de Scopus (ELSEVIER, 2020a), Google Acadêmico (GOOGLE, 2020) e InCites Journal Citation Reports (CLARIVATE ANALYTICS,2020b).

\section{ARTIGO}

CIT. (até Jan 2020)

H-index

FI (JCR)

PAUSTIAN, K.; LEHMANN, J.; OGLE, S.B.; REAY, D.; ROBERTSON, G.P.; SMITH, P. Climate-smart soils (Review). Nature, v. 532, Issue 7597, p. 49-57, 2016.

RICHARDS, D.R.; FRIESS, D.A. Rates and drivers of mangrove deforestation in Southeast Asia, 2000-2012. Proceedings of the National Academy of Sciences of the United States of America, v. 113, n. 2, p. 344-349, 2016.

OTTINGER, M.; CLAUSS, K.; KUENZER, C. Aquaculture: Relevance, distribution, impacts and spatial assessments - A review. Ocean and Coastal Management, v. 119, p. 244-266, 2016.
375

165
118 43.070

TIAN, B.; WU, W.; YANG, Z.; ZHOU, Y. Drivers, trends, and potential impacts of long-term coastal reclamation in China from 1985 to 2010. Estuarine, Coastal and Shelf Science, v. 170, p. 83-90, 2016.
WANG, X. et al. Assessment of soil erosion change and its relationships with land use/cover change in China from the end of the 1980s to 2010. Catena, v.137, p. 256268, 2016. quesito com o artigo mais citado. No geral, a média de citações é de 175 e de 5.671 JCR. Em relação ao H-Index a média é de 66, continuando próxima às da análise histórica (68) e da análise das publicações mais citadas dos últimos anos $(53,25)$. As três publicações envolvem pesquisadores do Instituto Nacional de Pesquisas Espaciais (INPE) com pesquisadores de institutos de pesquisa dos Estados Unidos.
Para obter base do escopo nacional foram analisadas as 3 publicações mais citadas das 36 publicações de pesquisadores brasileiros (Tabela 6).

Os artigos estão publicados em periódicos com alto JCR, sendo a revista "Remote sensing of environment" a liderar o
66

3851

Tabela 6 - Os três artigos nacionais mais citados de até 2019 com o número de citações, o maior h-index entre os autores e fator de impacto do periódico. Fonte: Compilado de Scopus (ELSEVIER, 2020a), Google Acadêmico (GOOGLE, 2020) e InCites Journal Citation Reports (CLARIVATE ANALYTICS,2020b).

\section{ARTIGO}

CIT.
(até Jan 2020)

91

\begin{abstract}
WESSELS, K.J.; DE FRIES, R.S.;, DEMPEWOLF, J.A; ANDERSON, L.O.; HANSEN, A.J.; POWELL, S.L.; MORAN, E.F. Mapping regional land cover with MODIS data for biological conservation: Examples from the Greater Yellowstone Ecosystem, USA and Pará State, Brazil. Remote Sensing of Environment, v 92, n. 1, p. 67-83, 2004.
\end{abstract} HESS, L.L.; MELACK, J.M.; AFFONSO, A.G.; BARBOSA, C.; GASTILBUHL, M.; NOVO, E.M. Wetlands of the Lowland Amazon Basin: Extent, Vegetative Cover, and Dual-season Inundated Area as Mapped with JERS-1 Synthetic Aperture Radar. Wetlands, v. 35, n. 4, p. 745-756, 2015. 


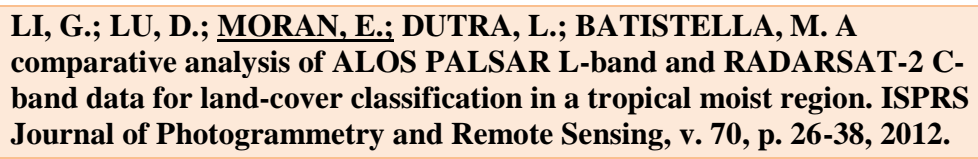

O trabalho desenvolvido por Hess et al. (2015) apresenta o primeiro mapeamento validado, moderadamente de alta resolução, da extensão das áreas úmidas, cobertura vegetal e estado de inundação de toda a bacia amazônica de planície. Pode ser considerado uma pesquisa de grande relevância num bioma pouco estudado com relação a áreas úmidas.

Li et al. (2012) aborda em sua pesquisa as dificuldades existentes no trabalho com dados de radar e a importância de combinar imagens radiométricas e texturais na classificação da cobertura do solo em regiões úmidas tropicais. Relatam que, no geral, os dados da banda $\mathrm{L}$ fornecem uma classificação muito melhor do que os dados da banda C. No entanto, nem os dados da banda $\mathrm{L}$ nem da banda $\mathrm{C}$ podem separar com precisão as classes detalhadas de floresta (por exemplo, floresta de terras altas, floresta inundável e floresta de cipós) ou sucessão (por exemplo, SS1, SS2 e SS3).

Por fim a pesquisa de Wessels et al. (2004) investigou a aplicação dos dados do MODIS para o mapeamento da cobertura territorial regional em resoluções moderadas ( 250 e $500 \mathrm{~m})$, para fins de conservação regional em duas áreas principais (Ecossistema Grande Yellowstone - GYE, EUA e o estado do
Pará, Brasil) usando dados MODIS e classificações em árvore de decisão. Segundo os autores, os mapas de cobertura da terra derivados do MODIS foram bem-sucedidos no mapeamento de tipos de cobertura extensos e menos no mapeamento de tipos de cobertura menores (por exemplo áreas úmidas e floresta decídua) que normalmente ocorrem em manchas menores que os pixels do MODIS.

\subsection{Representatividade dos softwares livres}

Por meio de refinamento sobre a string principal foram analisados os 1.585 artigos buscando-se saber quais desses faziam menção a software. Este primeiro refinamento resultou em 250 publicações. Nestes foram separados, por meio de um segundo refinamento, os que citavam utilizar software livre ou gratuito.

Retornaram 18 publicações onde notaram-se oito com os termos apenas nas referências bibliográficas, sendo que sete deles utilizavam software proprietário e três não especificavam os programas utilizados. Houve ainda um documento que não estava disponível para consulta. Por fim, apenas sete artigos dos 250 do primeiro refinamento utilizavam software livre (Figura 7).

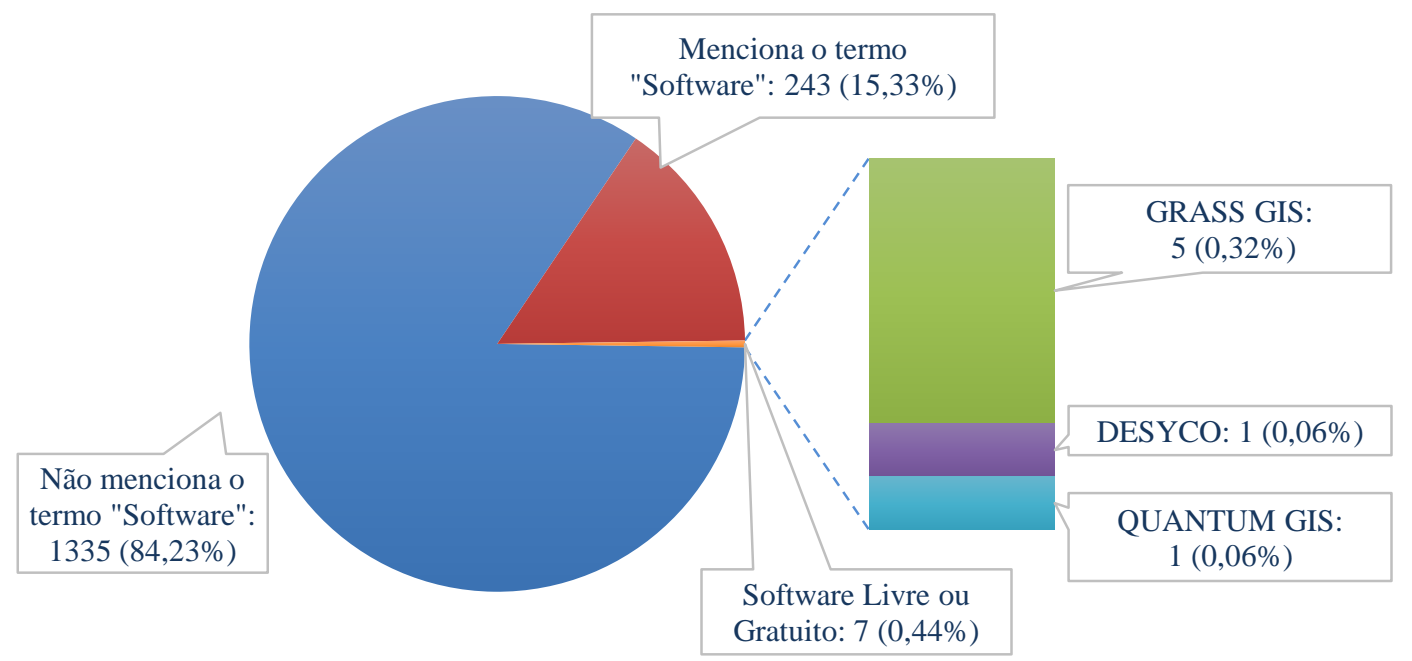

Figura 7 - Gráfico de representatividade dos softwares livres, onde apenas sete publicações das 1585 faziam o uso de programas livres. Fonte: Compilado de Scopus (ELSEVIER, 2020a).

Entre eles destaca-se o programa GRASS GIS (GRASS Development Team, 2017), sigla para Geographic Resources Analysis Support System (Sistema de Suporte a Análise de Recursos Geográficos), o software é gratuito e de código aberto e foi utilizado em cinco dos nove artigos encontrados.

\section{CONSIDERAÇÕES FINAIS}

As pesquisas acerca do uso e cobertura do solo em áreas úmidas com geotecnologias têm mantido um crescimento anual quase constante com uma média de citação considerável e crescimento exponencial que indica uma intensificação na discussão sobre o tema. 
De um modo geral, as publicações mais comuns são artigos científicos e artigos de revisão, com o inglês como a língua predominante. As publicações abrangem 23 áreas da ciência, com muitos documentos envolvendo múltiplas áreas.

Estados Unidos e China são os países com maior número de publicações e juntos possuem quase $50 \%$ dos artigos do tema. Também se destacam o Reino Unido com a maior média de citações por item e a Alemanha com mais relações de coautoria com outros países. O Brasil é o único país da América do Sul a figurar entre os 15 principais, estando em $10^{\circ}$ lugar do total de publicações. A relação de cooperação demonstra que boa parte das pesquisas envolve um número grande de pesquisadores de nacionalidades diversas.

Analisando as principais publicações em todo o período, destaca-se a presença de figuras-chave como orientador ou coordenador nos grupos de pesquisas. Estes possuem grande quantidade de publicações e $H$-index consideravelmente maior em relação aos demais. Porém ao analisar os últimos anos, notase uma mudança neste quadro com a presença de mais pesquisadores influentes em um mesmo grupo.

As principais publicações se referem principalmente a duas vertentes: uma sobre a criação, atualização e aperfeiçoamento de técnicas, métodos e bancos de dados, e a outra no tocante ao monitoramento e avaliação de possíveis impactos. A análise de rede com palavras-chaves apontou 'mudança no Uso do Solo' como mais recorrente conectada a 'Agricultura' indicando haver no tema uma grande parcela de artigos relativos ao avanço da área agrícola sob os recursos nativos. A grande reincidência no termo 'Landsat' a torna a possível principal imagem de satélite utilizada.

A baixa existência de registros sobre os softwares utilizados demonstrou pouca preocupação em reprodução posterior dessas pesquisas. A busca por softwares livres apresentou poucos resultados e mostrou ter ainda menos atenção em auxiliar a disseminação dessas pesquisas.

\section{REFERÊNCIAS}

CLARIVATE ANALYTICS. In Web of Science Core Collection. 2020a. Disponível em: <https://login.webofknowledge.com/>. Acesso em: 2 jan. 2020.

CLARIVATE ANALYTICS. In Cites Journal Citation Reports. 2020b. Disponível em: <https://jcr.clarivate.com/>. Acesso em: 3 jan. 2020.

Dewan, A. M.; Yamaguchi, Y. Land use and land cover change in Greater Dhaka, Bangladesh: Using remote sensing to promote sustainable urbanization. Applied geography, v. 29, 390-401, 2009.

ELSEVIER BV. In Scopus Database. 2020a. Disponível em: <https://www.scopus.com/>. Acesso em: 2 jan. 2020.

ELSEVIER BV. In Science Direct Database. 2020b. Disponível em: <https://www.sciencedirect.com/>. Acesso em: 2 jan. 2020 .

ELSEVIER BV. In Mendeley Desktop v1.19.3. 2020c. Disponível em: <https://www.mendeley.com/>. Acesso em: 2 jan. 2020.
França, A. M. DA S.; Sano, E. E. Influência de variações ambientais sobre o fluxo de $\mathrm{CO} 2$ em solos sob Campo Limpo Úmido no Cerrado. Sociedade e Natureza, v. 2, 197209, 2011.

Friedl, M. A. et al. MODIS Collection 5 global land cover: Algorithm refinements and characterization of new datasets. Remote sensing of Environment, v. 114, n. 1, 168-182, 2010.

Gardner, R. C.; Barchiesi, S.; Beltrame, C.; Finlayson, C. M.; Galewski, T.; Harrison, I.; Paganni, M.; Perennou, C.; Pritchard, D. E.; Rosenqvist, A.; Walpole, M. State of the World's Wetlands and their Services to People: A compilation of recent analyses. Ramsar Briefing Note, n. 7, $1-19,2015$.

GRASS Development Team, 2017. Geographic Resources Analysis Support System (GRASS) Software, Version 7.2. Open Source Geospatial Foundation. Electronic document:. http://grass.osgeo.org

GOOGLE. In Plataforma Google Acadêmico. Disponível em: <https://scholar.google.com.br/>. Acesso em: 3 jan. 2020.

Guo, M.; Li, J.; Sheng, C.; Xu, J.; Wu; L. A Review of Wetland Remote Sensing. Sensors, n. 17, 777, 2017.

Hewes, L. The northern wet prairie of the United States: Nature, sources of information, and extent. Annals of the Association of American Geographers, v. 41, n. 4, 307-323, 1951.

Kasischke, E. S.; Melack, J. M.; Dobson, M. C. The use of imaging radars for ecological applications - a review. Remote sensing of environment, v. 59, n. 2, 141-156, 1997.

Lee, K. H.; Lunetta, R. S. Wetland and environmental application of GIS. 1 ed. New York, USA: Lewis Publisher, 1996. 35p.

Lehner, B.; Doll, P. Development and validation of a global database of lakes, reservoirs and wetlands. Journal of Hydrology, v. 296, n. 1-4, 1-22, 2004.

Lopes, S.; Costa, M. T.; Fernández-Llimós, F.; Amante, M. J.; Lopes, P. F. A Bibliometria e a Avaliação da Produção Científica: indicadores e ferramentas. In: Actas do congresso Nacional de bibliotecários, arquivistas e documentalistas, $\mathrm{n}$. 11, 7, 2012.

Mao, G.; Liu, X.; Du, H.; Zuo, J.; Wang, L. Way forward for alternative energy research: A bibliometric analysis during 1994-2013. Renewable and Sustainable Energy Reviews, v. 48, 276-286, 2015.

Mitch, W. J.; Gosselink, J. G. Wetlands. 4 ed. New Jersey, EUA: Published by John Wiley \& Sons, 2007. 920p.

Nicholls, R. J. Coastal flooding and wetland loss in the 21st century: Changes under the SRES climate and socioeconomic scenarios. Glob. Environ. Chang., 14, 69-86, 2004. 
Olofsson, P.; Foody, G.; Herold, M.; Stehman; S. V.; Woodcock, C. E.; Wulder, M. A. Good practices for estimating area and assessing accuracy of land change. Remote Sensing of Environment, v. 148, 42-57, 2014.

Ottinger, M.; Clauss, K.; Kuenzer, C. Aquaculture: Relevance, distribution, impacts and spatial assessments - A review. Ocean and Coastal Management, v. 119, 244-266, 2016.

Ozesmi, S. L.; Bauer, M. E. Satellite remote sensing of wetlands. Wetlands ecology and management, v. 10, n. 5, 381-402, 2002.

Paustian, K.; Lehmann, J.; Ogle, S. B; Reay, D.; Robertson, G. P.; Smith, P. Climate-smart soils (Review). Nature, v. 532, n. 7597, 49-57, 2016.

Richards, D. R.; Friess, D. A. Rates and drivers of mangrove deforestation in Southeast Asia, 2000-2012. Proceedings of the National Academy of Sciences of the United States of America, v. 113, n. 2, 344-349, 2016.

Roth, N. E.; Allan, J. D.; Erickson, D. L. Landscape influences on stream biotic integrity assessed at multiple spatial scales. Landscape ecology, v. 11, n. 3, 141-156, 1996.

Tian, B.; Wu, W.; Yang, Z.; Zhou, Y. Drivers, trends, and potential impacts of long-term coastal reclamation in China from 1985 to 2010. Estuarine, Coastal and Shelf Science, v. 170, 83-90, 2016.

Van Eck, N. J.; Waltman, L. In Software VOSviewer. Centre for Science and Technology Studies. 2019. Disponível em: < https://www.vosviewer.com/>. Acesso em: 3 jan. 2020.

Walker, D. A. et al. The circumpolar Arctic vegetation map. Journal of Vegetation Science, v. 16, n. 3, 267-282, 2005.

Wang, X.; Zhao, X.; Zhang, Z.; Yi, L.; Zuo, L.; Wen, Q.; Liu, F.; $\mathrm{Xu}, \mathrm{J}$.; Hu, S.; Liu, B. Assessment of soil erosion change and its relationships with land use/cover change in China from the end of the 1980s to 2010. Catena, v.137, 256-268, 2016.

Wood Júnior, T.; De Medeiros Costa, C.C. Avaliação do impacto da produção científica de programas selecionados de pósgraduação em Administração por meio do índice H. Revista de Administração, v. 50, n. 3, 325-337, 2015.

Yuan, F.; Sawaya, K. E.; Loeffelhoz, B. C.; Bauer, M.E. Land cover classification and change analysis of the Twin Cities Metropolitan Area by multitemporal Landsat remote sensing. Remote sensing of Environment, v. 98, n. 2-3, 317328, 2005.

Zhang, P.; Yan, F.; Du, C. A comprehensive analysis of energy management strategies for hybrid electric vehicles based on bibliometrics. Renewable and Sustainable Energy Reviews, n. $48,88-104,2015$.

\section{AGRADECIMENTOS}

Os autores agradecem ao PGTA/UFMS (Programa de PósGraduação em Tecnologias Ambientais), da Universidade Federal de Mato Grosso do Sul - UFMS. Ao PGRN (Programa de Pós-Graduação em Recursos Naturais, da da Universidade Federal de Mato Grosso do Sul - UFMS.

O presente trabalho foi realizado com apoio da Coordenação de Aperfeiçoamento de Pessoal de Nível Superior - Brasil (CAPES) - Código de Financiamento 88887.494036/2020-00;

Agradecemos ainda a CAPES pelo acesso ao Portal de Periódicos.

Ao PNPD-CAPES pela bolsa de Pós-Doutorado 2017-2018 a Eliane Guaraldo;

Ao $\mathrm{CNPq}$ (Conselho Nacional de Desenvolvimento Científico e Tecnológico) pela bolsa de produtividade em pesquisa de A. C. Paranhos Filho (CNPq Processo 305013/2018$1)$.

O presente trabalho foi realizado com apoio da Fundação Universidade Federal de Mato Grosso do Sul - UFMS/MEC Brasil.

Recebido em: 13/05/2020

Aceito para publicação em: 18/11/2020 